\title{
An interlaboratory quality control programme for the measurement of tuberculosis drugs
}

\author{
To the Editor:
}

Tuberculosis (TB) remains a major global health problem. Inadequate exposure to TB drugs constitutes one of the main factors underlying suboptimal treatment response and development of resistance, as evidenced by results from: the in vitro hollow fibre model [1]; clinical studies on relationships between drug concentrations and response [2-5]; a pharmacogenetic trial [6]; and a recent meta-analysis [7].

More specifically, these concentration-effect evaluations suggest that clinicians should prescribe higher doses of rifampicin $[2,5]$, that acetylator status should be used to determine the dose of isoniazid $[6,7]$, and that higher doses and exposures to pyrazinamide may increase efficacy $[4,5]$.

Clearly, such studies also underline the relevance of careful concentration-effect evaluations during the development of new TB drugs that will eventually be applied by clinicians all over the world.

Finally, these studies support the concept of therapeutic drug monitoring (TDM) of TB drugs as applied by clinicians and pharmacists in selected centres around the world [8-10]. In contrast to administering the same fixed dose to all patients, TDM seeks to individualise drug doses, guided by measurement of serum (or plasma) drug concentrations.

Both pharmacokinetic evaluations in academia and in drug development programmes, and the use of TDM require the application of analytical methods for TB drugs. Intralaboratory (internal) method validation and intralaboratory quality control (QC) procedures, such as validation of equipment and qualification of technicians, should ensure that these methods have sufficient accuracy, precision and specificity. In addition, participation in an interlaboratory (external) QC (or proficiency testing) programme is an essential component of quality assurance and also provides evidence of laboratory competence for clinicians, researchers, accrediting bodies and regulatory agencies [11].

It is worth noting that (semi-)automatic immuno-assays are not available for the measurement of TB drugs. TB drugs are measured by so-called chromatographic assays that have to be developed and validated within each laboratory, sometimes based on published analytical methods, which also explains the current limited availability of TDM for TB drugs. Clearly, more complex, labour-intensive assays developed "in-house" hinder the inter-laboratory comparability of drug measurements and definitely require participation in an external QC programme.

Unfortunately, an international interlaboratory QC programme is as yet unavailable for TB drugs. We therefore started such an external QC programme, focussing on first-line as well as several second-line TB drugs.

TB drugs involved in the first round of the QC programme were isoniazid, rifampicin, pyrazinamide, ethambutol (first-line TB drugs), moxifloxacin and linezolid (second-line TB drugs). All raw drug substances were of analytical quality and had a high and specified purity ( $>99 \%)$. TB drugs were weighed out on independently calibrated balances and dissolved in methanol/water using calibrated pipettes and volumetric flasks. Drug-free human serum was obtained from the regional blood bank. Blank serum was spiked with solutions of TB drugs to obtain two QC samples. Each of the two samples contained all six TB drugs in either low or intermediate/high concentrations (table 1). The QC samples were immediately freeze-dried in view of the instability of some of the drugs and to decrease shipping costs. We had previously assessed stability under various conditions after freeze-drying (unpublished data). All weighed-in concentrations were considered as true values.

Seven laboratories from two continents participated in the first round of the programme. The freeze-dried QC samples were transported at room temperature to these laboratories. The laboratories were informed that the samples had to be stored at $4-8^{\circ} \mathrm{C}$ after receipt. They were requested to reconstitute the samples with $1 \mathrm{~mL}$ water and to analyse them as soon as possible. Reconstituted samples could also be stored at 


\begin{tabular}{|c|c|c|c|c|c|c|}
\hline Drug & Measurements $\mathrm{n}$ & Conc. ${ }^{\#}$ & $\begin{array}{c}\text { Measured conc. } \\
\text { relative to true value } \\
\%\end{array}$ & Absolute inaccuracy \%" & $\mathrm{n}$ & $\%$ \\
\hline Isoniazid & 7 & Low & 95 (84-105) & $5(3-16)$ & 7 & 100 \\
\hline Rifampicin & 6 & Int./high & 87 (49-135) & $27(1-51)$ & 2 & \\
\hline \multirow[t]{2}{*}{ Pyrazinamide } & 5 & Low & $108(104-117)$ & $8(4-17)$ & 5 & 100 \\
\hline & 5 & Int./high & $110(101-118)$ & $10(1-18)$ & 5 & \\
\hline \multirow[t]{2}{*}{ Ethambutol } & 6 & Low & $107(75-137)$ & $14(4-37)$ & 4 & 75 \\
\hline & 6 & Int./high & $104(98-142)$ & $4(1-42)$ & 5 & \\
\hline Moxifloxacin & 3 & Low & 98 (95-108) & $5(2-8)$ & 3 & 100 \\
\hline
\end{tabular}

Data are presented as median (range) unless otherwise stated. Conc.: concentration; int.: intermediate. \#: low and int./high conc. levels were 0.48 and $6.47 \mathrm{mg} \cdot \mathrm{L}^{-1}$ (isoniazid), 1.41 and $5.60 \mathrm{mg} \cdot \mathrm{L}^{-1}$ (rifampicin), 18.79 and $58.61 \mathrm{mg} \cdot \mathrm{L}^{-1}$ (pyrazinamide), 0.93 and $4.78 \mathrm{mg} \cdot \mathrm{L}^{-1}$ (ethambutol), 0.31 and $2.65 \mathrm{mg} \cdot \mathrm{L}^{-1}$ (moxifloxacin), and 0.91 and $11.09 \mathrm{mg} \cdot \mathrm{L}^{-1}$ (linezolid). ${ }^{9}$ : inaccuracy is the percentage bias from the true conc., i.e. inaccuracy $=(100 \times$ measured conc./true conc. $)-100 \%{ }^{+}$: acceptable measurements are within $20 \%$ of the true conc.

$-80^{\circ} \mathrm{C}$ for analysis at a later time point. The participating laboratories were asked to return their results within 6 weeks of dispatch of the samples.

Descriptive statistics were calculated after standardisation of all laboratory results to percentages with reference to the true value. By subtracting $100 \%$ from these percentages, the percentage bias from the true concentration (inaccuracy) was calculated. 20\% limits around the true values were considered to be appropriate threshold values for satisfactory measurements, as used and justified in our other international QC programmes [12,13]. All participants were informed about their performance within 2 months of reporting their results.

Two of the seven participating laboratories were able to measure all six TB drugs; one laboratory measured five drugs (all four first-line TB drugs and moxifloxacin), two laboratories measured four drugs (all four first-line TB drugs, but not the second-line drugs moxifloxacin and linezolid) and the two remaining laboratories were able to measure less than four of the first-line drugs. The analytical methodology used was conventional high-performance liquid chromatography, ultra-performance liquid chromatography, liquid chromatography with mass spectrometric detection or gas chromatography with mass spectrometric detection.

A total of 58 analyses were performed, with satisfactory (accurate) results reported for 48 (83\%) measurements. Table 1 summarises the results by TB drug. Rifampicin concentrations were too low in three laboratories, both at the low and medium/high concentration. Furthermore, the programme showed ethambutol concentrations that were too high for one laboratory, a trend for higher concentrations of pyrazinamide in one participating laboratory, and similarly high concentrations of rifampicin in another laboratory.

The concentration level did not appear to affect accuracy, as 24 (83\%) out of 29 of the measurements was performed satisfactorily both at the low and intermediate/high concentration levels.

When arranged by performing laboratory, it appeared that three out of seven laboratories performed all measurements within $20 \%$ inaccuracy limits (i.e. a $100 \%$ score of 12 out of 12,10 out of 10 and two out of two accurate results). The other laboratories measured 10 out of $12(83 \%)$, four out of six (67\%), five out of eight $(63 \%)$ and five out of eight $(63 \%)$ of the concentrations within the accepted limits.

$83 \%$ of the measurements were within $20 \%$ limits of the true weighed-in concentrations, meaning the initial results of this QC programme show a reasonably good performance of the participating laboratories in measuring TB drugs. For comparison, first rounds of similar programmes for antiretroviral drugs and antifungal drugs yielded satisfactory measurements in $65 \%$ and $77 \%$ of the measurements, respectively $[12,13]$. One out of six (17\%) measurements of TB drugs was still inaccurate and such figures are relevant in the context of pharmacokinetic studies and TDM. In this respect, it should be recognised that the first round of this programme may have selected the best-performing laboratories or those with emphasis on quality assurance. 
The results for rifampicin in this first round were clearly worse than for other drugs. We have considered the possibility that these results might be due to incomplete dissolution of the freeze-dried samples, as rifampicin is slightly soluble in aquous solutions. For the next rounds we will validate the dissolution of the samples once more.

Beyond the possible QC programme problems, many other sources of error may explain the rifampicin results, including method (validation), equipment, technical and clerical problems, as well as unexplained errors [11]. In the past we have evaluated such possible sources of error in other QC programmes by sending an error evaluation form to the laboratories; this will be considered for future rounds of the TB programme.

It is worth noting that low rifampicin plasma/serum concentrations have been reported by many authors in populations all over the world. Based on the results of this first round of the QC programme, it cannot be excluded that some of these findings may be explained by inaccuracy in the measurement of rifampicin, yet the results of the first round of this programme should not be overinterpreted and results from subsequent rounds should be awaited.

Many research consortia are currently studying the effects of higher doses of rifampicin, and the resulting higher exposures achieved with these doses, both in pulmonary and extrapulmonary TB. This emphasises the relevance of accurate assays for rifampicin and the usefulness of this QC programme.

In addition to the low rifampicin concentrations measured in some laboratories, the programme revealed possible inaccuracies or previously undetected problems relating to the analysis of ethambutol and pyrazinamide in some laboratories. By participating in the programme, these laboratories were alerted to these problems, and this may enable and incite them to optimise their methods and intralaboratory QC procedures. Clearly, this is how external QC control testing aims to achieve improvement in laboratory performance and attain interconvertibility of results between laboratories $[9,11]$.

For the first round of the programme, we selected all first-line TB drugs (group I of TB drugs), moxifloxacin as a pivotal representative from group III of TB drugs (fluoroquinolones) [14] and linezolid because it is frequently used for multidrug-resistant/extensively drug-resistant TB treatment despite its allocation in group $\mathrm{V}$ of TB drugs $[10,15]$. In future rounds of the programme, we will consider incorporating other TB drugs (with consideration of the requests of the participants) and offer two patient cases in relation to the measurements, for those involved in TDM for TB drugs. An increase in the number of participants may allow for evaluation of the effects of the drug measured, the concentration level or the analytical methodology on the accuracy of the results and will provide a better impression of overall laboratory performance.

In summary, external QC for measurement of TB drugs in serum/plasma is pivotal considering the high level of interest in the pharmacokinetics of TB drugs, both for the development of new TB regimens and for direct application in patient care (TDM). We have developed an interlaboratory (external) QC programme or proficiency testing programme for TB drugs and invite laboratories to participate in this programme.

0 @ERSpublications

Quality control in TB drug measurement is essential for the development of new drugs and for patient care http://ow.ly/KxWNc

Rob E. Aarnoutse ${ }^{1,2}$, Marieke G.G. Sturkenboom ${ }^{3}$, Karen Robijns ${ }^{2}$, Anneke R. Harteveld ${ }^{2}$, Ben Greijdanus ${ }^{3}$, Donald R.A. Uges ${ }^{3}$, Daniel J. Touw ${ }^{2,3}$ and Jan-Willem Alffenaar ${ }^{3}$

${ }^{1}$ Dept of Pharmacy, Radboud University Medical Center, Nijmegen, The Netherlands. ${ }^{2}$ Association for Quality Assessment in TDM and Clinical Toxicology (KKGT) (a section of the Dutch Foundation for Quality Assessment in Medical Laboratories (SKML)), The Hague, The Netherlands. ${ }^{3}$ Dept of Clinical Pharmacy and Pharmacology, University of Groningen, University Medical Center Groningen, Groningen, The Netherlands.

Correspondence: Rob E. Aarnoutse, Dept of Pharmacy, Radboud University Medical Center, PO Box 9101, 6525 HB, Nijmegen, The Netherlands. E-mail: Rob.Aarnoutse@radboudumc.nl

Received: Sept 262014 | Accepted after revision: Feb 092015 | First published online: April 162015

Conflict of interest: Disclosures can found alongside the online version of this article at erj.ersjournals.com

\section{References}

1 Srivastava S, Pasipanodya JG, Meek C, et al. Multidrug-resistant tuberculosis not due to noncompliance but to between-patient pharmacokinetic variability. J Infect Dis 2011; 204: 1951-1959.

2 Mehta JB, Shantaveerapa H, Byrd RPJr, et al. Utility of rifampin blood levels in the treatment and follow-up of active pulmonary tuberculosis in patients who were slow to respond to routine directly observed therapy. Chest 2001; 120: 1520-1524.

3 Weiner $\mathrm{M}$, Benator $\mathrm{D}$, Burman $\mathrm{W}$, et al. Association between acquired rifamycin resistance and the pharmacokinetics of rifabutin and isoniazid among patients with HIV and tuberculosis. Clin Infect Dis 2005; 40: 1481-1491. 
4 Chideya S, Winston CA, Peloquin CA, et al. Isoniazid, rifampin, ethambutol, and pyrazinamide pharmacokinetics and treatment outcomes among a predominantly HIV-infected cohort of adults with tuberculosis from Botswana. Clin Infect Dis 2009; 48: 1685-1694.

5 Pasipanodya JG, McIlleron H, Burger A, et al. Serum drug concentrations predictive of pulmonary tuberculosis outcomes. J Infect Dis 2013; 208: 1464-1473.

6 Azuma J, Ohno M, Kubota R, et al. NAT2 genotype guided regimen reduces isoniazid-induced liver injury and early treatment failure in the 6-month four-drug standard treatment of tuberculosis: a randomized controlled trial for pharmacogenetics-based therapy. Eur J Clin Pharmacol 2013; 69: 1091-1101.

7 Pasipanodya JG, Srivastava S, Gumbo T. Meta-analysis of clinical studies supports the pharmacokinetic variability hypothesis for acquired drug resistance and failure of antituberculosis therapy. Clin Infect Dis 2012; 55: 169-177.

8 Alsultan A, Peloquin CA. Therapeutic drug monitoring in the treatment of tuberculosis: an update. Drugs 2014; 74: 839-854.

9 Srivastava S, Peloquin CA, Sotgiu G, et al. Therapeutic drug management: is it the future of multidrug-resistant tuberculosis treatment? Eur Respir J 2013; 42: 1449-1453.

10 Lange C, Abubakar I, Alffenaar JW, et al. Management of patients with multidrug-resistant/extensively drug-resistant tuberculosis in Europe: a TBNET consensus statement. Eur Respir J 2014; 44: 23-63.

11 Clinical Laboratory and Standards Institute. Using Proficiency Testing to Improve the Clinical Laboratory; Approved Guideline. 2nd Edn. Wayne, Clinical Laboratory and Standards Institute, 2007.

12 Aarnoutse RE, Verweij-van Wissen CP, van Ewijk-Beneken Kolmer EW, et al. International interlaboratory quality control program for measurement of antiretroviral drugs in plasma. Antimicrob Agents Chemother 2002; 46: $884-886$.

13 Brüggemann RJ, Touw DJ, Aarnoutse RE, et al. International interlaboratory proficiency testing program for measurement of azole antifungal plasma concentrations. Antimicrob Agents Chemother 2009; 53: 303-305.

14 Pranger AD, van Altena R, Aarnoutse RE, et al. Evaluation of moxifloxacin for the treatment of tuberculosis: 3 years of experience. Eur Respir J 2011; 38: 888-894.

15 Sotgiu G, Centis R, D'Ambrosio L, et al. Efficacy, safety and tolerability of linezolid containing regimens in treating MDR-TB and XDR-TB: systematic review and meta-analysis. Eur Respir J 2012; 40: 1430-1442.

\section{Linezolid for multidrug-resistant tuberculosis in HIV-infected and -uninfected patients}

To the Editor:

Two recent systematic reviews found that treatment outcomes with regimens containing linezolid for complicated cases of multidrug-resistant (MDR) tuberculosis (TB) are equal to or better than those reported for uncomplicated MDR-TB $[1,2]$ and better than those reported among patients treated for extensively drug-resistant (XDR)-TB [3-6]. Existing data on treatment outcomes with linezolid for MDR-TB are predominantly among HIV-uninfected patients; $<10 \%$ of patients were HIV co-infected in the reviews $[1,2]$. There is concern over the safety of using linezolid within MDR-TB regimens for $\mathrm{HIV}$-infected patients due to underlying HIV-related neuropathy and bone marrow dysfunction as well as a potentially higher incidence of adverse events in patients on antiretroviral therapy (ART), notably anaemia with zidovudine and peripheral neuropathy with stavudine [7]. Given the high rate of HIV/TB co-infection in many settings, we report here our clinical experience with the use of linezolid for treatment of XDR-TB and complicated MDR-TB among HIV-infected and -uninfected patients in community-based programmes supported by Médecins Sans Frontières (MSF) in two high-burden settings in Khayelitsha, South Africa [8], and Mumbai, India [7]. We carried out a retrospective analysis of routinely collected data. Programmes at both sites were approved by either MSF or local university ethics review boards. Due to restricted access to linezolid in South Africa as well as limited capacity for operational research in both programmes, the numbers are small, and data were collected and analysed over a limited time period only.

At time of analysis in August 2013, 34 patients (17 from each site); one with MDR-TB, 16 with pre-XDR-TB (defined as MDR plus resistance to either a fluoroquinolone or an injectable agent) and 17 with XDR-TB) had received individualised regimens with a median of eight drugs (range 7-10), including linezolid, for $\geqslant 1$ month. 18 (53\%) patients started treatment on initial detection of second-line drug resistance; the remainder only after second-line TB treatment appeared to be failing due to continued culture positivity or reconversion from negative to positive. The median second-line treatment duration 